\title{
Globalization of Dietary Wild Foods Protect against Cardiovascular Disease and all Cause Mortalities? A Scientific Satement from the Interna- tional College of Cardiology, Columbus Paradigm Institute and the Inter- national College of Nutrition
}

\author{
RB Singh $^{1, *}$, Fabien DeMeester ${ }^{2}$ Daniel Pella ${ }^{3}$, Tapan K Basu ${ }^{4}$ and Ronald Ross Watson ${ }^{5}$
}

\author{
${ }^{1}$ President, International College of Cardiology, Halberg Hospital and Research Institute, Moradabad-10(UP)244001, India; \\ ${ }^{2}$ President, Columbus Paradigm Institute, Waterloo, Belgium; \\ ${ }^{3}$ Executive Director, International College of Cardiology, Faculty of Medicine, Safaric University, Kosice, Slovakia; \\ ${ }^{4}$ Executive Director, International College of Nutrition, Department of Agricultural, Food and Nutritional Science, \\ University of Alberta, Edmonton, Canada; \\ ${ }^{5}$ Director, Division of Health Promotion Sciences, Mel and Enid Zuckerman College of Public Health, and School \\ of Medicine, University of Arizona, Arizona, Tucson, USA
}

\begin{abstract}
Dietary pattern consisting of high intakes of vegetables, fruits, legumes, fish, poultry, and whole grains, can be protective against mortality from cardiovascular and malignant diseases. A meta-analysis of prospective cohort studies demonstrated that an increased consumption of fruit and vegetables from less than 3 to more than 5 servings/day is related to a $17 \%$ reduction, whereas an increased intake from 3 to 5 servings/day is associated with a smaller and borderline significant reduction in CAD risk. These results provide evidence supporting that 5 or more servings per day of fruit and vegetables, are needed to protect from CVD. Other cohort studies identified 2 dietary patterns from data collected on serial food frequency questionnaires. One pattern, called "prudent", was characterized by a high vegetables, fruit, legumes, fish, poultry, and whole grains. The other pattern, which is proatherogenic, called "Western", corresponded to a high consumption of red meat, processed meat, refined grains, french fries, sweets, and desserts. More recently, "Designer" foods containg nutrients such as w-3 fatty acids, selected groups of vitamins and trace elements, and antioxidants are being available that may have the potential for inclusions in the "prudent" dietary pattern in the future. Indeed in the year 2009, European Union has allocated a budget of 9 million Euro for conducting research to examine the effects of "designer" foods on health biomarkers. These efforts are being recognized by the World Health Organization, International College of Nutrition and the International College of Cardiology.
\end{abstract}

\section{INTRODUCTION}

Recently, food consumption patterns have been proposed to be important in the pathogenesis of non-communicable diseases and all cause mortality [1-3]. According to a prospective cohort study [1], a dietary pattern consisting of high intakes of vegetables, fruits, legumes, fish, poultry, and whole grains can be protective against mortality from cardiovascular and malignant diseases. These findings are in parallel with the outcomes of several epidemiological and intervention studies, reporting inverse relationship between consumption of fruits and vegetables and the incidence of coronary artery disease (CAD) [2-8]. Nowson and his associates quantitatively assessed the relation between fruit and vegetable intake and incidence of CAD by carrying out a meta-analysis of cohort studies which reported relative risks (RRs) and corresponding 95\% confidence interval (CI) of CAD with respect to frequency of fruit and vegetable intake [2]. These studies, included a total of 278, 459 individuals

*Address correspondence to this author at the International College of Cardiology, Halberg Hospital and Research Institute, Moradabad-10(UP) 244001, India; E-mail: icn2005@sancharnet.in,drkk@ dataone.in
(9143 CAD events) with a median follow-up of 11 years. The individuals who had less than 3 servings/day of fruit and vegetables, the pooled RR of CAD was 0.93 (95\% CI: $0.86-$ $\left.1.00, \mathrm{P}^{1} / 40.06\right)$ while those with more than 5 servings/ day , the RR was $0.83(0.77-0.89, \mathrm{P}<0.0001)$. This meta-analysis of prospective cohort studies demonstrated that an increased consumption of fruit and vegetables, from less than 3 to more than 5 servings/day is related to a $17 \%$ reduction, whereas an increased intake from 3 to 5 servings/day is associated with a smaller and borderline significant reduction in CAD risk. These results provide evidence supporting that 5 or more servings per day of fruit and vegetables, are needed to protect from CVD.

In a large, prospective, observational study [1] involving 72,113 female nurses who were free of CAD, stroke, diabetes, and cancer, factor analysis identified 2 dietary patterns from data collected on serial food frequency questionnaires. One pattern, called "prudent", was characterized by a high consumption of vegetables, fruit, legumes, fish, poultry, and whole grains. The other pattern, called "Western", corresponded to a high consumption of red meat, processed meat, refined grains, french fries, sweets, and desserts. Individuals 
were classified by their level of adherence to both the prudent diet and the Western diet. After baseline data collection in 1984, a follow-up study lasted for 18 years, during which time 6011 deaths occurred; of these 3319 (52\%) as a result of cancer; 1154 (19\%) from cardiovascular disease (CVD); and 1718 (29\%) from other causes. In multivariable analyses, there was a $17 \%$ lower risk of total mortality among those who were most adherent to the prudent diet (highest versus lowest quintile of adherence), a $28 \%$ lower risk of CVD mortality, and 30\% lower mortality from non-CVD, non-cancer causes. Interestingly, there was no significant relationship between the prudent dietary pattern and cancer. A comparison of the highest and lowest quintiles of adherence showed that consumption of the Western diet was associated with increased total mortality (21\%), CVD mortality (22\%), cancer mortality (16\%), and mortality from non-CVD, noncancer causes $(31 \%)$. Hence, except for cancer, risk relationships for the prudent and Western dietary patterns appear to be the inverse of each other: Mortality thus was increased as adherence to the prudent diet decreased and adherence to the Western diet increased.

The relationship between dietary patterns and risk of acute coronary syndrome (ACS) was examined in a standardized case-control study INTERHEART, involving participants from 52 countries [10]. Using the principlecomponent analysis technique, the authors identified 3 major dietary patterns; Oriental (high intake of tofu and soy and other sauces), Western (high in fried foods, salty snacks, eggs, and meat), and prudent (high in fruit and vegetables). Consistent with previous studies in single within-population cohort studies, the authors found an inverse association between the prudent pattern score and risk of ACS and a significant positive association between the Western pattern score and increased risk of ACS. The Oriental pattern was not significantly associated with risk. The investigators constructed a dietary risk score based on 7 food items on the food-frequency questionnaire (meat, salty snacks, fried foods, fruits, green leafy vegetables, cooked vegetables, and other raw vegetables) and found that a higher score (indicating a poor diet) was strongly associated with ACS risk: Those in the highest quartile of the score had nearly 2 -fold increased risk, even after adjustment for established coronary risk factors. In sensitivity analyses, the investigators found a consistent association for the composite diet score between men and women and across different regions of the world (North America, western Europe, Australia, central Europe, Middle East, Africa, south Asia, southeast Asia, China, and South America). On the basis of an arbitrary cut-off point of the score (top 3 quartiles versus the bottom quartile), the investigators estimated that $30 \%$ of MI could be explained by unhealthy diets worldwide.

Although Western-style changes in food patterns are widely believed to adversely influence risk of chronic diseases, few epidemiological studies have directly linked dietary patterns and mortality from coronary heart disease. The INTERHEART study was the first large study to quantify eating patterns in all geographic regions of the world. It provides evidence that despite different food habits in various populations, reproducible patterns can be found in diverse regions of the world. These findings are important because there has been a concern that dietary patterns derived through a data-driven approach such as principle-component analysis may be highly unstable and nonreproducible because of very different eating habits in different populations.

In recent years, dietary-patterning analysis has been increasingly used as an alternative method to traditional singlenutrient analysis because it can assess cumulative effects of the overall nutrients in a diet. Habitual intake patterns are typically quantified by statistical methods such as factor or cluster analysis or diet-quality indices based on prevailing dietary recommendations or healthful traditional diets (eg, the Mediterranian and Indo-Mediterranian diets). Principlecomponent analysis is commonly used to define dietary patterns using food consumption information to identify common underlying dimensions (factors or patterns) of food intake. The method aggregates specific food items based on the degree to which these food items are correlated with each other. A summary score for each pattern is then derived and can be used to examine relationships between various eating patterns and outcomes of interest such as coronary heart disease and other chronic diseases. Previous validation studies found that 2 major patterns (the prudent and Western patterns) identified through principle-component analysis of food consumption data assessed by food frequency questionnaires were reproducible over time and correlated reasonably well with the patterns identified from diet records.

The consistent association observed between the Western or unhealthy dietary pattern (high in animal products, salty snacks, refined starches and sugar and fried foods and low in fruits and vegetables) and ACS risk in different regions of the world from the INTERHEART study and other studies, provides some evidence of the adverse effects of globalization on human nutrition and chronic disease risk, but the evidence is indirect because these studies did not specifically assess the impact of global trade and marketing on food consumption patterns across different countries $[1,9,12]$. Despite this weakness, most recent studies suggest that the current trend of dietary convergence toward a typical Western diet is likely to play a role in the globalization of obesity, CVD, diabetes and cancer.

Fetuin-A, a protein almost exclusively secreted by the liver, induces insulin resistance and subclinical inflammation in rodents. Circulating fetuin-A levels are elevated in humans with metabolic syndrome and insulin resistance, indicating that this circulating protein may have nutritional origin. The association between fetuin- $\mathrm{A}$ levels and the risk of future ACS and ischemic stroke (IS) were studied in a case-cohort study(12) based on the European Prospective Investigation into Cancer and Nutrition Potsdam Study comprising 27, 548 middle-aged subjects of the general population. Fetuin-A levels were measured in plasma of individuals who developed $\operatorname{ACS}(n=227)$, IS $(n=168)$, and in individuals who remained free of cardiovascular events $(n=2198)$ during a follow-up of $8.2 \pm 2.2$ years. Individuals in the highest compared with the lowest quintile of plasma fetuin-A had significantly increased risks of ACS (relative risk, 3.80; 95\% confidence interval, 2.37 to $6.10 ; P$ for trend $<0.0001)$ and ischemic stroke (relative risk, 3.93; 95\% confidence interval, 2.17 to $7.12 ; P$ for trend $<0.0001$ ) after adjustment for sex and age. Additional adjustment for smoking status, body mass index, waist circumference, alcohol consumption, educational attainment, physical activity, hypertension, diabetes 
mellitus, total and high-density lipoprotein cholesterol, and C-reactive protein attenuated these risks, but only moderately (MI: relative risk, 3.25; 95\% confidence interval, 2.01 to 5.28 ; $P$ for trend $<0.0001$; IS: relative risk, 3.78 ; $95 \%$ confidence interval, 2.06 to $6.94 ; P$ for trend $<0.0001)$. These data provide evidence for a link between high plasma fetuinA levels and an increased risk of MI and IS. Therefore, more research is warranted to determine the role of fetuin-A in the pathophysiology of cardiovascular disease. It is possible that fetuin $\mathrm{A}$ is related to diet and lifestyle factors and w-6/w-3 ratio in a diet, which should be studied among these patients. It could be a manifestation of pre-metabolic syndrome with increased risk of vascular variability disorders.

\section{WHAT ARE THE CHALLENGES}

The main challenges include availability of prudent wild foods at reasonable cost, time taken in preparation, taste and aggressive publicity of western foods by the food industry. Food industry is not commonly interested in preparing health foods that are known to decrease mortality and morbidity; their primary goal is to sell products at a competitive price for profitability. Wild foods such as fish are increasingly being scarced.

We are now in a situation where the public health policies are clear for recommending prudent dietary patterns and providing health foods to community. However, the food policies of the government and of the farming and food industries are geared to completely different goals. The growing demands on the farmers and the food industry for a healthier diet are now being recognized, as marketing opportunity for developing healthy foodstuffs, with balanced amount of fatty acids, low glycemic foods and other nutrients in the diet.

It is a paramount of importance that World Health Organization and Food and Agriculture Organization together with the international scientific societies like World Heart Federation, International College of Cardiology, American Heart Association, European Society of Cardiology, International College of Nutrition and International Union of Nutritional Sciences open doors to collaborate with Columbus Paradigm Institute, Waterloo, Belgium (www.columbusconcept.com), to formulate guidelines for manufacturing of health foods. This non-profit organization has expertized how to develop wild type of foods for prevention of diseases and enhance high quality of productive life. These foods are rich in micronutrients, antioxidants, vitamins and minerals, while providing high $\mathrm{w}-3$ fatty acids, monounsaturated fatty acids, proteins and slowly absorbed carbohydrates. Longterm, randomized, controlled clinical trials are necessary to provide a scientific proof regarding the safety and efficacy of these foods in the prevention of diseases. These trials should be supported by the government agencies and international organizations such as European Union grants, because private companies are incapable in providing adequate research funds, necessary for such trials. European Union has sanctioned in its 2008-09 budget, Euro 1.5 million for development of functional food and ingrdient(call: FP7KBBE-2009-3) which is very encouraging.

In one trial, [3], it has been demonstrated that whole grain- enriched hypocaloric diet is superior to refined grain group in decreasing risk of CVD in patients with metabolic syndrome. However, no study has referred to w-6/w-3 fatty acid ratio of the two diets, which could have been a more important factor for decreasing inflammation due to increased content of w-3 fat and low w-6 fat [4]. Wild-whole grains rich in w-3 fatty acids, antioxidants and magnesium may provide even better results compared to whole grain grown by modern farming. Prior to the Agricultural Revolution, our diet was based on wild plants, eggs, fish and meat from wild animals, whereas today, greatest percentage of food supply is contributed by cereal grains, (mainly refined), refined carbohydrates and meat from farm sources. These foods are rich in w-6 fatty acids and low in w-3 fatty acids, fiber,phytoestrogens, minerals and antioxidants. CVD, diabetes mellitus, cancer, autoimmune diseases, rheumatoid arthritis, asthma and depression are associated with increased production of thromboxane A2, leucotrienes, interleukins-1 and 6 , tumor necrosis factor-alpha and C-reactive proteins. Increased dietary intake of w-6 fatty acids is known to enhance all these risk factors as well as atherogenicity of cholesterol which have adverse proinflammatry effects resulting into thrombosis and acute coronary syndrome (ACS) [3-8].

ACS may be due to thrombosis in the unstable plaque, and associated with activation of neurohormones and proinflammatory cytokines; interleukin- 6 , and 18 , resulting into neuroendocrine dysfunction, which worsens the prognosis in these patients [4-8]. There may be oxidative stress, hyperglycemia, hyperinsulinemia, hypertriglyceridemia, increased levels of catecholamines, free fatty acids and cortisol which may cause endothelial dysfunction and rupture of plaque resulting in to recurrent ACS [4-8]

Recent studies [4-8, 13, 14], indicate that eating high fat, refined carbohydrate rich fast foods(western diet), can produce a similar proinflammatory state in our body, with increase in oxidative stress, free fatty acids and triglycerides; resulting into endothelial dysfunction, which may have adverse effects in patients with ACS [4-8]. It is therefore, logical to avoid western diet in patients with ACS, and to administer Indo-Mediterranean foods which may be beneficial to vascular endothelium and myocardium [2-8]. There is no precise and proven guideline for dietary advice in patients with ACS, which may be protective against recurrent cardiac events. A Mediterranean soup, which is made of tomatoes, grapes/raisins, vegetables; carrot, spinach, walnuts, almonds+lin/chia seeds and olive oil or yogurt containing, walnuts, almonds, raisins, could be prepared for ready use, for nonpharmacological intervention, among patients of ACS.These foods appear to be protective against metabolic syndrome [3]. Such recepies have been commonly used in the Indo-Mediterranean diet heart study and Indian experiment of infarct survival [6-8]. A diet containing 400g/day of fruits and vegetables along with $400 \mathrm{~g} /$ day of legumes and other whole grains, and $50 \mathrm{~g} /$ day of almonds and walnuts, in conjunction with $25-50 \mathrm{~g} /$ day of Columbus oil(olive oil 88 $\%+$ flax seed oil 12.0\%; P:S=w-6:w-3:=1:1 Patent Application WO2005020698) may be protective against all cause mortality and morbidity. Upto one egg daily has not been shown to have any adverse effects on coronary and proinflammatory factors. Hence, an egg in a breakfast along with Mediterranean soup as a substitution of refined starches and meat, may be a prudent choice in the primary prevention of CVD. Designer eggs with modified fatty acid composition 
and increased content of w-3 fatty acids appear to be good for prevention of metabolic syndrome (4).

It would be interesting to know how many patients died of ACS in various cohort studies [1, 2, 9-12], prudent diet verses western diet. The dietary intakes in most of these cohort studies are reported by self reported questionnaires and hence subject to bias; a sample size may ignore this weakness of such cohort studies [1, 2, 12]. Most researchers studying dietary patterns do not mention the nutrient content of their prudent diet, [1, 2, 10-12]; w-3 fatty acid is one such nutrient, which is rich in fruits, vegetables, nuts and whole grains. It would be interesting to know the role of refined starches and sugar, large meals, decreased intake of fruits, vegetables, whole grains and nuts and serum nitrite levels as risk predictors of ACS in these cohorts [1, 2, 12]. Fruits, vegetables, nuts, whole grains, animal foods rich in w-3 fatty acids are slowly absorbed and may prevent the increase in free fatty acids, and inflammation, which is a characteristic of Columbus foods(www.columbus-concept.com) and therefore such foods may decrease the risk in ACS as well as metabolic syndrome [3-8, 13, 14]. Omega-3 fatty acids can regulate leptin gene expression and the concentrations of anandamides in the brain, which in turn binds to endogenous cannabinoid receptors and regulate food intake and satiety and weight gain.

It seems that suboptimal nutrition is an important factor in the global burden of CAD. Cohort studies [1, 2, 9-12] add to this evidence by identifying the association between an unhealthy dietary pattern and risk of ACS in 52 countries around the world and among women in the United States [1, $2,12]$. By defining a common feature of nutrition transitions with their accompanying risks to public health, the authors underscore the importance of developing an effective and comprehensive set of health policies that address globalization and its impact on obesity and diet-related chronic diseases. Indeed, the findings from recent studies indicate [3-8, $13,14]$ that a common set of dietary recommendations based on availability of wild type of foods can be made to prevent chronic diseases on a worldwide basis. These foods are known to have anti-inflammatory effects and can modulate about 125 genes, most of them proinflammatory, which express due to increased consumption of western meals [13, 14]. Such a public health strategy should emphasize replacing saturated, trans, and w-6 fats with unsaturated fats from natural vegetable oils rich in w-3 fatty acids (canola oil, rapeseed oil), and replacing refined grain products, starches and sugar with whole grain products, legumes, fruits, and vegetables, fish and nuts [3-8]. International College of Cardiology and International College of Nutrition in association with Columbus Paradigm Institute is developing guidelines to include wild type of foods in the dietary patterns which may be highly protective in the prevention of morbidity and mortality due to CVD, diabetes and cancer.

\section{REFERENCES}

[1] Heidemann C, Schulze MB, Franco OH, et al. Dietary patterns and risk of mortality from cardiovascular disease, cancer, and all causes in a prospective cohort of women. Circulation 2008; 118: 230-7.

[2] He FJ, Nowson CA, Lucas M, MacGregor GA. Increased consumption of fruit and vegetables is related to a reduced risk of coronary heart disease: meta-analysis of cohort studies. J Hum Hypertens 2007; 21: 717-28.

[3] Katcher HI, Legro RS, Kunselman AR, et al. The effects of whole grain- enriched hypocaloric diet on cardiovascular disease risk factors in men and women with metabolic syndrome. Am J Clin Nutr 2008; 87: 79-90.

[4] Singh RB, DeMeester F, Mechirova V, Pella D, Otsuka K. Fatty acids in the causation and therapy of metabolic syndrome. In wild type foods in health promotion and disease prevention. In: DeMeester WF, Watson RR, Eds. Humana Press, NJ 2008; pp. 263-84.

[5] De Logeril M, Salen P, Martin JL, et al. Mediterranean diet, traditional risk factors and the rate of cardiovascular complications after myocardial infarction. Final report of the Lyon Diet Heart Study. Circulation 1999; 99: 779-85.

[6] Singh RB, Dubnov G, Niaz MA, et al. Effect of an IndoMediterranean diet on progression of coronary disease in high risk patients: a randomized single blind trial. Lancet 2002; 360: 145561.

[7] Singh RB, Rastogi SS, Verma R, Bolaki L, Singh R. An Indian experiment with nutritional modulation in acute myocardial infarction. Am J Cardiol 1992; 69: 879-85.

[8] Singh RB, Pella D, DeMeester F. What to eat and chew in acute myocardial infarction. Eur Heart J 2006; 27: 1628-29.

[9] Hu FB. Globalization of food patterns and cardiovascular disease risk. Circulation 2008; 118: 1913-4.

[10] Iqbal R, Anand S, Ounpuu S, et al. Dietary patterns and the risk of acute myocardial infarction in 52 countries. Circulation 2008; 118 : 1929-37.

[11] Appel LJ. Dietary patterns and longevity. Circulation 2008; 118 : 214-15.

[12] Weikert C, Stefan N, Shulze MB, et al. Plasma fetuin-A levels and the risk of myocardial infarction and ischemic stroke. Circulation 2008; 118: 2555-62.

[13] Vogel RA. Eating vascular biology and atherosclerosis: a lot to chew on. Eur Heart J 2006; 27: 13-4.

[14] Esposito K, Giugiliano D. Diet and inflammation: a link to metabolic and cardiovascular diseases. Eur Heart J 2006; 27: 15-20. 